\title{
COURRIER
}

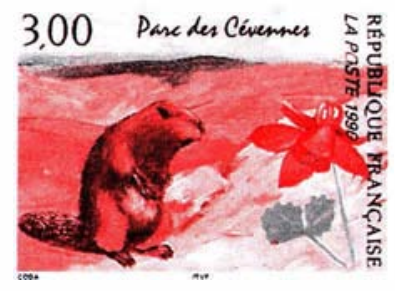

\section{Devons-nous introduire des considérations éthiques dans la nomenclature médicale?}

Nombreuses sont les maladies génétiques portant le nom du ou des médecins qui en firent la première description. Ces désignations éponymiques perdurent, même dans les banques de données informatiques, accessibles sur Internet, comme dans OMIM (Online Mendelian Inheritance in Man). Consacrées par l'usage et le temps, elles définissent, mieux que toute autre dénomination, certaines affections : comment désigner autrement la myopathie de Duchenne, la chorée de Huntington ou la maladie d'Alzheimer ? Et parce que leur nom est lié à une maladie, on connaît même parfois la vie de ces hommes. Les femmes, elles, sont bien rares dans ce palmarès mandarinal! Évoquons seulement, pour le plaisir, Julia Bell - du syndrome de Martin-Bell, aujourd'hui maladie de l'X fragile -, célibataire invétérée qui devint centenaire et continua à porter intérêt à la génétique jusqu'à sa mort. Guillaume Duchenne naquit à Boulogne, d'une famille de marins, mais peu que Peter Becker, né à Hambourg, servit dans l'armée de l'air allemande lors de la dernière guerre.

Parfois un héros se cache derrière le médecin, tel Johannes Pompe, résistant hollandais qui avait un émetteur-radio sont certes anachroniques, mais elles

Beaucoup d'entre nous savent que clandestin dans son laboratoire et fut exécuté par les nazis, à 44 ans, juste avant la libération des Pays-Bas, en 1945 [1]. Que sa mémoire soit ainsi honorée semble équitable.

Mais en revanche, que faire quand on découvre, derrière des éponymes, de véritables criminels ? C'est l'interrogation que lance Peter Harper [2] à propos de Julius Hallervorden et de Hugo Spatz. Ces deux neuropathologistes, qui décrivirent vers 1922 une nouvelle forme de dégénérescence cérébrale familiale avec lésions histologiques caractéristiques, semblent avoir collaboré en toute conscience à l'œuvre de destruction des handicapés mentaux entreprise dans l'Allemagne hitlérienne. Grâce à la proximité d'un hôpital psychiatrique fonctionnant alors comme centre d'extermination, ils purent disposer de centaines de cerveaux parfaitement fixés, ce qui leur permit de poursuivre leurs recherches et d'accroitre la renommée de l'institut (Kaiser Wilhelm Institut) où ils avaient des postes de responsabilité qu'ils conservèrent, sans être inquiétés, jusqu'à leur retraite. Leurs noms furent toutefois mentionnés lors d'une cérémonie commémorative en l'honneur des victimes du nazisme dont le compte rendu parut dans un journal de neuropathologie [3] ainsi que dans le livre "Science nazie, science de mort " [4].
Faut-il attendre que le défaut moléculaire de cette maladie soit découvert pour changer son nom ? Doit $\bullet$ dès maintenant substituer à ces sinistres éponymes le terme de «dystrophie neuroaxonale "? Ou au contraire, comme le souhaite Benno Muller-Hill [4], est-il préférable de conserver délibérément ces noms afin de se souvenir des événements des années noires ?

Ces questions, que Peter Harper a bien fait de poser à l'ensemble de la communauté scientifique, concernent aussi naturellement les lecteurs de médecine/ sciences et pourraient susciter, je crois, de leur part, une réflexion

\section{Simone Gilgenkrantz}

\section{RÉFÉRENCES}

1. Beighton P, Beighton G. The man behind the syndrome. Berlin : Springer Verlag, 1982. 2. Harper PS. Lancet 1996; 348: 1224-5.

3. Pfeiffer B. Brain Pathol 1991 ; 1 : 125-31. 4. Muller-Hill B. Science nazie, science de mort. Paris: Odile Jacob, 1989.
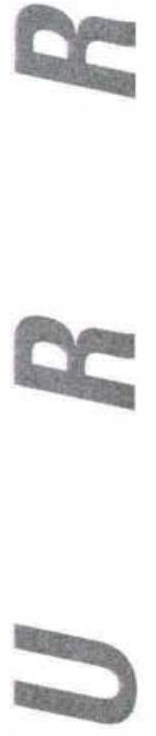


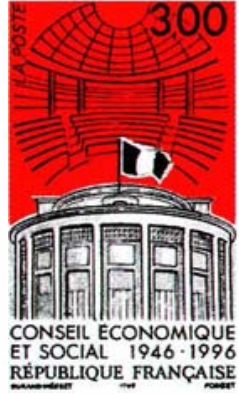

\section{Il ne faut pas confondre protéine canalaire et co-transporteur}

Le titre de la nouvelle "Canalopathies rénales: le gène du syndrome de Bartter néonatal " - paru dans $\mathrm{m} / \mathrm{s}$ $n^{\circ} 10$, vol.12, p. 1168 - induit le lecteur en erreur. La note porte essentiellement sur des mutations trouvées dans le gène codant pour le co-transporteur $\mathrm{Na}-\mathrm{K}-2 \mathrm{Cl}$ chez les malades atteints de syndrome de Bartter néonatal et fait référence aux mutations du gène du co-transporteur $\mathrm{Na}-\mathrm{Cl}$ impliquées dans la maladie de Gitelman, ainsi qu'aux mutations du canal sodium épithélial impliquées dans la maladie de Liddle. Le mot "canalopathie " doit être restreint aux mutations des protéines canalaires. Les exemples les plus connus des maladies génétiques de ce type sont la mucoviscidose (mutation dans le canal chlorure-CFTR), la myotonie congénitale (mutation dans le canal chlorure dépendant du voltage, $\mathrm{ClC} 1$ ), la paralysie périodique hypokaliémique (canal calcium sensible à la dihydropyridine) ou encore la maladie de Liddle, citée à juste titre dans la note.

Il existe une différence fondamentale dans les mécanismes de transport d'ions par un canal ionique et par un co-transporteur. Le canal ionique est une protéine membranaire qui transporte les ions à travers une membrane selon leurs gradients électrochimiques. Il est admis que la partie transmembranaire de la pro- sélectivité varie d'un canal à l'autre (par exemple, canaux cationiques, anioniques...). Le transport couplé (ou secondairement actif) est effectué par une protéine transmembranaire qui permet le transport d'un ion (une molécule) contre son gradient électrochimique grâce à l'énergie dissipée par la translocation simultanée d'un autre ion selon son gradient électrochimique favorable (exemple : co-transporteurs $\mathrm{Na}-\mathrm{K}-2 \mathrm{Cl}$, $\mathrm{Na}-\mathrm{Cl}$, Na-glucose...). Les mécanismes moléculaires responsables de ce type de transport sont encore mal connus. Une des hypothèses actuellement à l'étude suppose la présence sur la protéine du co-transporteur de sites de reconnaissance de l'ion sodium ( $\mathrm{m} / \mathrm{s} n^{\circ} 10$, vol. $\left.12, p .1136\right)$. Une autre différence entre un canal et un co-transporteur est le flux d'ions transportés. Il est admis que la plupart des canaux transportent environ $10^{6}$ à $10^{7}$ ions $/ \mathrm{s}$ contre seulement $10^{4}$ à $10^{5}$ ions/s pour les transports couplés. En conclusion, les mécanismes moléculaires et thermodynamiques du transport ionique par des protéines canalaires et des transporteurs couplés sont différents.

\section{Aleksander Edelman}

Inserm U. 467. Faculté de médecine Necker-Enfants Malades 156, rue de Vaugirard, 75730 Paris Cedex 15, France.

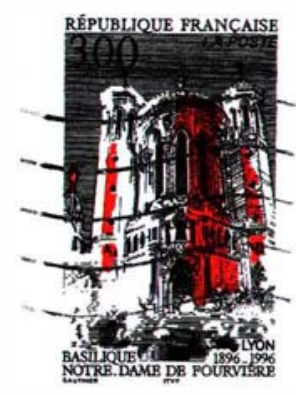

Diagnostic génétique pré-implantatoire (DPI) : réponse à un éditorial

Nos commentaires [1] à la suite de l'article de Viville et al. [2] ont inspiré une réaction de Pierre Jouannet [3] qui nous oblige à préciser la position que nous développons depuis plusieurs années $[4,5]$.

Ecrire, comme P. Jouannet, que «la maîtrise in vitro de la fécondation... conduit obligatoirement à se préoccuper de la qualité des embryons ainsi conçus ", c'est énoncer une évidence pour en faire une stratégie.

Evidence: ne pas transférer un embryon non viable (par exemple polyspermisque); stratégie: identifier tous les signes de moindre "qualité " dans un projet sélectif. Tout le problème du DPI est dans cette nuance que P. Jouannet évacue en rappelant que le «tri embryonnaire sur critères génétiques et morphologiques est pratiqué dans tous les laboratoires de FIV ». Ce faisant, il assimile le constat de non-viabilité avec le risque de maladie héréditaire puisque le tri couramment pratiqué en FIV consiste à éliminer les embryons polyploïdes (plus de 2 pronoyaux visibles), lesquels sont incapables d'évoluer en enfant vivant. Puis $P$. Jouannet confond eugénisme avec sexisme en développant le thème du choix du sexe des enfants. Rappelons que l'eugénisme se propose d'améliorer l'espèce, ce qui ne peut se faire 
en éliminant l'un des deux sexes. A l'issue de chacune de ses "démonstrations", l'éditorialiste prend son propre discours en défaut et paraît admettre que le débat est ailleurs. Que n'y va-t-il d'emblée?

A la suite de Viville et al., P. Jouannet prétend que le DPI n'a "jamais été utilisé pour des raisons de convenance". Nous avons évoqué le recours au DPI pour éviter les risques d'hémophilie, d'iso-immunisation Rhésus, ou de cancer colorectal. Pour un "type de diagnostic encore peu répandu", voilà une avancée bien rapide depuis les indications initiales (myopathie, mucoviscidose...) et il ne reste qu'à connaître le délai ( 5 ans, 10 ans, 30 ans ?) qui nous sépare du DPI contre les risques d'asthme, d'obésité, ou de myopie. Bien sûr, ce pronostic s'appuie plutôt "sur des convictions et des affirmations à l'emporte-pièce que sur des faits", comme il arrive toujours quand on tente de décrire l'avenir plutôt que le passé. Mais, pas plus que les autres partisans du DPI, P. Jouannet n'avance de propositions de garde-fous contre les dérives prévisibles du DPI. Comment définir les graves maladies héréditaires pour les différencier des affections moins graves, puis des petits défauts? Comment éviter que le DPI, proposé chez tel couple pour éviter une maladie grave, ne profite de la présence simultanée de nombreux embryons pour élargir son champ au dépistage de moindres défauts? A l'hypothèse de la pente glissante, comment "substituer celle de l'escalier" si on ne peut pas en décrire les marches sur une échelle de gravité?

Quand P. Jouannet s'indigne de notre opposition au DPI, "contraire à toute tradition en matière de recherche et de pratique médicale ", il néglige les bouleversements, pas seulement techniques, introduits dans la médecine par la pratique de l'assistance médicale à la procréation; a u point qu'une loi a paru nécessaire pour en contrôler les développements, définir des compétences, exiger des bilans d'activité, bref, pour rompre avec la tradition... Finalement P. Jouannet nous donne en exemple «l'expérience de la commission de génétique de la fédération des CECOS qui traite des sujets du même ordre dans le domaine du don de gamètes». Pourtant, le DPI intervient comme une garantie alors que le conseil génétique prétend informer sur un risque; là où le premier permet de sélectionner la «qualité » parmi de nombreux embryons, le second joue en amont, sur la décision de procréer, ou bien en aval, sur celle d'avorter. Il est, par ailleurs, malvenu de citer cet exemple pour illustrer "l'aide à la décision" des patients quand la même commission des CECOS s'arroge l'énorme privilège d' "apparier des couples reproducteurs" hors de toute information ou de toute demande des patients. En ce sens la sélection de donneurs de sperme, puis leur "appariement" avec des femmes receveuses, en fonction de facteurs de risque objectivés par des experts, est bien l'acte fondateur de cet eugénisme moderne, savant et bienveillant, qui ne peut que s'épanouir avec le DPI [6]. L'exigence du produit "sans faute", par l'IAD comme par le DPI, sera toujours en retard d'un gène juste identifié ou d'un virus soudainement apparu; plus encore, elle se heurtera sans cesse à l'imperfection constitutive de l'élu, donneur de sperme ou embryon.

Une façon de contourner le vrai problème du DPI est de poser de fausses questions, comme celle qui titre l'éditorial [3] : "Peut-on intégrer l'embryon humain dans le champ médical et scientifique?»... Bien sûr, on le peut, mais la question n'est pas là. Elle porte sur l'issue prévisible de certaines avancées de la technoscience, que son objet soit l'embryon, le fœtus, l'enfant, ou l'adulte. L'embryon ne mérite certainement pas plus de respect que la personne née, mais il constitue un levier incomparablement plus puissant pour altérer l'humanité, serait-il confié aux mains médicales les plus respectables et les plus compétentes

\section{RÉFÉRENCES}

1. Testart J, Sèle B. Le diagnostic préimplantatoire n'est pas un diagnostic prénatal précoce. Med Sci 1996; 12: 1398-401.

2. Viville J, Ray P, Wittemer C, Ohl J, Dellenbach $\mathrm{P}$, Gerlinger P. Diagnostic génétique préimplantatoire: legislation et aspects éthiques. Med Sci 1996; 12: 1394-7.

3. Jouannet P. Peut-on intégrer l'embryon humain dans le champ médical et scientifique? Med Sci 1996; 12: 1331-3.

4. Testart J. Le désir du gène. Paris: Flammarion 1995: 286 $\mathrm{p}$.

5. Testarı J, Sèle B. Towards an efficient medical eugenics: is the desirable always the feasible? Hum Reprod 1995 ; 10: 3086-90.

6. Testart J. La procréation médicalisée. Collection Dominos. Paris: Flammarion, 1993: 77-9.

\section{Jacques Testart}

Directeur de Recherche à l'Inserm, Unité Inserm U. 355, 32, rue des Carnets, 92140 Clamart, France.

\section{Bernard Sèle}

Professeur à la faculté de médecine de Grenoble, Inserm U. 309, Groupe de biologie de la reproduction, Faculté de médecine, 38700 La Tronche, France.

\section{$\mathrm{m} / \mathrm{S}$}

